\title{
Features of the ISO-25498: Method of Selected Area Electron Diffraction Analysis in Transmission Electron Microscopy
}

\author{
Delu Liu* \\ Department of Materials Physics, University of Science and Technology Beijing, No.30, Xueyuan Road, \\ Beijing 100083, China
}

\begin{abstract}
International standard ISO-25498 specifies the method of selected area electron diffraction (SAED) analysis in TEM. It is applicable to the acquisition of SAED patterns, indexing the patterns and calibration of diffraction constant. Several features of this standard are introduced. As an example of the applications, phosphide with nanometer scale in a low-carbon steel produced by compact strip production process was analyzed by SAED and EDX. The phosphide precipitates in the steel are identified as $\mathrm{M}_{x} \mathrm{P}$, where $x$ is $2-3$ and $\mathrm{M}$ is $\mathrm{Fe}, \mathrm{Ti}, \mathrm{Cr}$, or Ni. It possesses a hexagonal lattice with lattice parameter $a=0.609 \mathrm{~nm}$ and $c=0.351 \mathrm{~nm}$.
\end{abstract}

Key words: international standard, electron diffraction, TEM, phosphide precipitate, CSP steels, EDS microanalysis, nanoparticles

\section{INTRODUCTION}

The international standard ISO-25498 (published in 2010) specifies the method of selected area electron diffraction (SAED) analysis in TEM. This standard was prepared by Technical Committee ISO/TC 202 (microbeam analysis)/SC3 (analytical electron microscopy). Experts from nine countries of the member bodies have participated in the work.

The standard ISO-25498 is applicable to the acquisition of SAED patterns from crystalline specimens, indexing the patterns and calibration of diffraction constant. It is also a supplementary technique for the acquisition of highresolution images, microdiffraction, or convergent beam diffraction studies. The present contribution introduces some features of ISO-25498 briefly. An example of the applications is also given.

\section{Materials and Methods}

As an example of the applications, phosphide $\mathrm{M}_{x} \mathrm{P}$ particles were analyzed by SAED and EDX. Composition of the experimental steel is: (mass\%) C: $0.06, \mathrm{Si}: \leq 0.6, \mathrm{Mn}: \leq 0.6$, $\mathrm{S}: \leq 0.01, \mathrm{P}: \leq 0.12, \mathrm{Cu}: \leq 0.3, \mathrm{Cr}: \leq 0.5, \mathrm{Ni}: \leq 0.2, \mathrm{Ti}: \sim 0.13$. The steels were produced by compact strip production (CSP) processing. Specimens were extractive powder, which were extracted electrochemically from the steel then spread on the microgrids.

\section{Features of the ISO-25498}

Optimizing the Optical Coupling of TEM for SAED Analysis In a TEM, diffraction pattern appears in the back focal plane of the objective lens (OL). This pattern is magnified by the intermediate and projector lenses, and displayed on a

() MICROSCOPY SOCIETY OF AMERICA 2013

${ }^{\star}$ Corresponding author. E-mail: deluliu@sina.com viewing screen. The interest area is selected by the field limiting aperture (FLA), which is inserted at the imaging plane of the OL. Therefore, this specimen area is actually selected from its image. In SAED working mode, the object plane of the intermediate lens (IL) coincides with back focal plane of the OL, but in the imaging mode this object plane of the IL coincides with the imaging plane of the OL (see Fig. 1). Discrepancy of the selected area may arise from spherical aberration (coefficient $C_{\mathrm{s}}$ ) of the $\mathrm{OL}$ and the specimen height. The selected area in the specimen will be shifted by distance $y$ :

$$
y=C_{\mathrm{s}}\left(2 \theta_{\mathrm{B}}\right)^{3}+2 \theta_{\mathrm{B}} \cdot \Delta f
$$

where $\Delta f$ is the deviation of the specimen height from the focusing position and $\theta_{\mathrm{B}}$ is Bragg angle of the reflection beam.

Thus, the diffraction information actually comes from a specimen area, which is obviously larger than the area constrained by the FLA aperture. Objects out of the selected area may also have contribution to the SAED pattern. It can be seen that the specimen height is critical to ensure that a diffraction pattern is corresponding to the area selected by the FLA aperture. The minimum analyzable area for the SAED method is limited by OL aberration. The attempt of the operating procedure described in ISO-25498 is to optimize optical coupling of these lenses, FLA aperture, and the specimen. Then credible results could be received.

\section{Obtain Information from Three-Dimensional Space} of the Specimen

Information from three-dimensional space of the specimen is essential for diffraction study. As a single diffraction pattern consisting of only zero Laue zone will provide only planar (two-dimensional) information. It is necessary to acquire additional diffraction patterns from the same area but with different crystal orientation or from different grains/ 


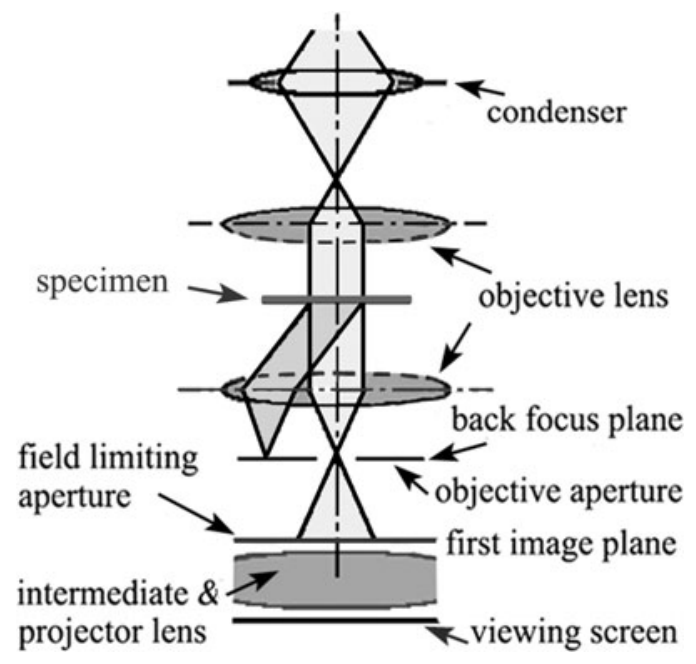

Figure 1. Illustration of the lens matching for selected area electron diffraction working condition.

particles of the same phase. Two methods, the specimen tilting and the multicolony procedure, are specified by the standard.

1. The specimen tilting procedure: choose a row of closespaced diffraction spots collinear with the central spot on the diffraction pattern. Align this row with the tilting axis and obtain the second (and more) diffraction pattern by tilting the specimen.

2. The multicolony procedure: acquire the second or more patterns from several areas, i.e., different particles or grains of the same phase. In this situation, it is necessary to choose appropriate diffraction spots to form a darkfield image to confirm the source of each diffraction pattern. Further confirmation may be achieved through simultaneous analysis of EDX.

The three-dimensional reciprocal space of a specimen may be constructed by these patterns.

\section{Indexing Diffraction Pattern by the Characteristic Parallelogram}

The success of the SAED method relies on the validity of indexing the diffraction patterns. Concept of the characteristic parallelogram is introduced into ISO-25498 for indexing of the patterns (Edington, 1975; Guo et al., 1983). A diffraction pattern consisting of only zero Laue zone from a single crystal appears as an array of "spots," the basic unit of which is characterized by a basic parallelogram (Fig. 2). This parallelogram is constituted by the vectors $\mathbf{R}_{1}$ and $\mathbf{R}_{2}$. These vectors are corresponding to the nearest and next nearest diffraction spots $h_{1} k_{1} l_{1}$ and $h_{2} k_{2} l_{2}$ to the central spot, respectively, in the pattern. Work out the interplanar spacing corresponding to the diffraction spots $h_{1} k_{1} l_{1}$ and $h_{2} k_{2} l_{2}$ in the pattern. Then the diffraction pattern can be indexed by comparing with the characteristic parallelogram and the zone axis can be decided. The method of indexing the diffraction pattern by using the characteristic parallelogram is recommended by ISO-25498.

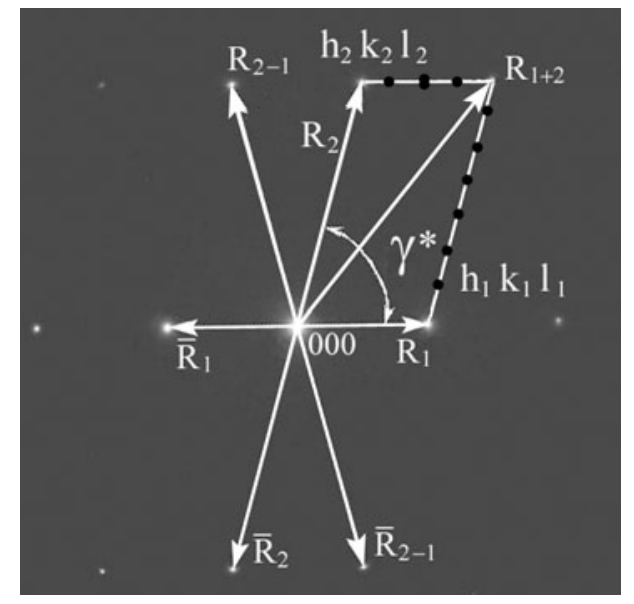

Figure 2. An example of the spot pattern showing the characteristic parallelogram constituted by $\mathbf{R}_{1}$ and $\mathbf{R}_{2}$.

\section{Improvement in Accuracy of the SAED Studies}

After a SAED pattern has been obtained and indexed correctly, a successful analysis also depends on the fact that the interplanar spacing of each reflection in the pattern can be measured properly. This measurement involves the use of a reference material so that the camera constant can be determined.

Sources of error in determining interplanar spacing include fluctuations in the voltage supply and lens current of the imaging lenses, shift in specimen height, and the aberration from the lenses. The effects of these parameters are discussed in ISO-25498. Provided the experimental conditions for acquiring the diffraction pattern of the standard and the unknown specimen are completely identical, the effects of variation in microscope parameters are very small. Thus, the main errors will come from measurement of the diffraction pattern. Effects of the lens aberration, especially the astigmatism, are crucial. In ISO-25498, the procedure for determination of the camera constant and the interplanar spacing is specified to minimize the errors.

\section{Identification of Phosphide $\mathrm{M}_{x} \mathrm{P}$ by SAED and EDX Analysis}

As the solidification rate and cooling rate of the steels in the CSP processing is highly rapid, supersaturation of the elements such as Ti, O, S, N, P, etc. is much increased in the steels. Precipitation behavior of the second phases can be varied obviously in the steels (Liu, 2009). A large number of precipitates with nanometers in size such as sulfides, oxides, and carbonitrides were observed. However, phosphide particles with nanometer scale in low-carbon steels are seldom reported.

Recently, phosphide precipitates in the experimental steel have been investigated by SAED and EDX (Lou \& Liu, 2010). The results showed that phosphide particles $M_{x} P$ $(x=2-3)$ in nanometer scale exist in the steels. The value of $x$ is $2-3$; metallic elements $\mathrm{M}$ are $\mathrm{Fe}, \mathrm{Ti}, \mathrm{Cr}$, or Ni. Figure 3 shows a TEM image of a rod-shaped phosphide particle (Fe, $\mathrm{Ti})_{x} \mathrm{P}$. The EDX and SAED patterns of the particle are given 


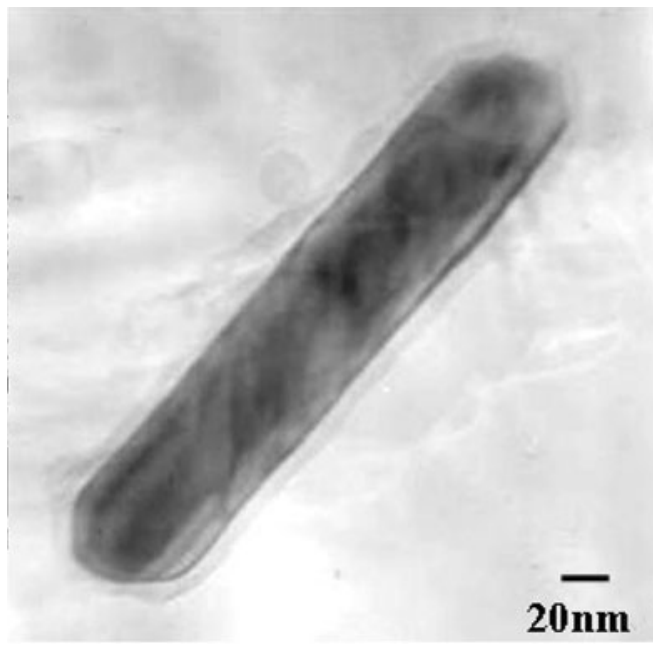

Figure 3. Transmission electron microscopic image of a $\mathrm{M}_{x} \mathrm{P}$ particle in the compact strip production steel (incident beam direction is $[010]$ of the particle).

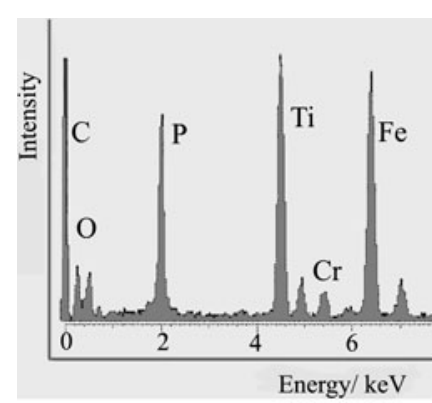

(a)

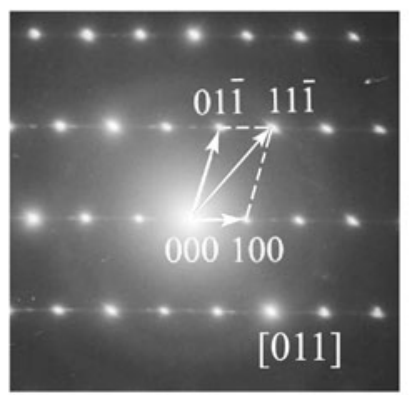

(c)

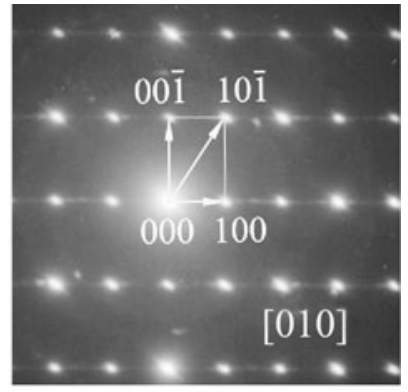

(b)

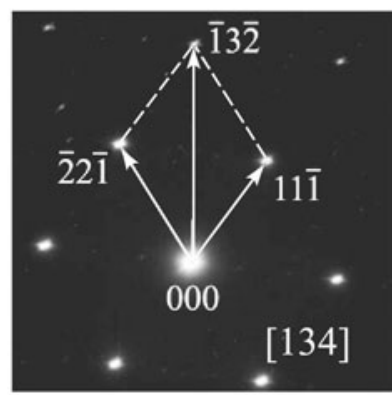

(d)
Figure 4. EDS and selected area electron diffraction (SAED) patterns of the rod-shaped phosphide $\mathrm{M}_{x} \mathrm{P}$ particle in Figure 3. (a) EDS of the particle; (b) SAED pattern of [010] zone axis; (c) SAED pattern of [011] zone axis; (d) SAED pattern of [134] zone axis.

in Figure 4. It is pointed out by SAED study that the phosphide possesses a hexagonal structure with lattice parameters $a=0.609 \mathrm{~nm}, c=0.351 \mathrm{~nm}$. This is consistent with the structure of phosphide $(\mathrm{Cr}, \mathrm{Fe}, \mathrm{Ni}, \mathrm{Ti})_{2} \mathrm{P}$ (namely, $\mathrm{M}_{2} \mathrm{P}$ ) in an austenite stainless steel (Vodarek et al., 1988).

\section{DiscusSIONS}

It is the first time to publish an international standard for SAED analysis on TEM. Only fundamental procedures are specified in this standard. Several important phenom- ena of the electron diffraction study such as second diffraction, higher Laue zone diffraction, etc. are not involved in the standard ISO-25498 (2010). However, complicated diffraction patterns are frequently encountered in material studies. Therefore, efficacious diffraction study relies on comprehensive understanding of the microscopy principle and crystallography.

Although some standard diffraction spot patterns for cubic systems are given in the annex of ISO-25498, it is suggested that the diffraction patterns be calculated in advance by a program to solve the diffraction patterns. In practice, this is more reliable and also convenient, especially for those crystals with lower symmetry.

\section{SUMMARY}

The ISO-25498 (2010) is the first standard for SAED analysis in TEM. Only fundamental procedures are specified in this standard. Further improvement is in prospect. All of the applications are based on the fact that the SAED patterns are correctly acquired, indexed, and measured. Correct operation for the electron diffraction study relies on comprehensive understanding of the microscopy principle.

Phosphide $\mathrm{M}_{x} \mathrm{P}(x=2-3)$ precipitates in nanometer scale in the CSP steel containing titanium and phosphor are identified by SAED and EDX. The results approved that the phosphide $\mathrm{M}_{x} \mathrm{P}$ can present as dispersive precipitates in the steels produced by CSP process.

\section{ACKNOWLEDGMENTS}

This work was supported by a grant from SAC (Standardization Administration of the People's Republic of China). The specimen preparation and experimental investigation was also carried out by Dr. Lou Yanzhi, Master Bai Mingzhuo, and Master Ni Xiaoqing at the University of Science and Technology Beijing.

\section{REFERENCES}

Edington, J.W. (1975). Practical Electron Microscopy in Materials Science, Vol. II, Electron Diffraction in The Electron Microscope. London: Macmillan \& Co Ltd.

Guo, K., Ye, H. \& Wu, Y. (1983). Application of Electron Diffraction Patterns in Crystallography. China: Science Press.

ISO-25498 (2010). Microbeam Analysis-Analytical Electron Microscopy-Method of Selected Area Electron Diffraction Analysis in Transmission Electron Microscopes. Geneva, Switzerland: International Organization for Standardization.

LIu, D. (2009). Precipitations in the CSP low carbon steels. In Ultra-Fine Grained Steels, Yuqing, W. (Ed.), pp. 190-228. Beijing: Metallurgical Industry Press; Berlin, Heidelberg, New York: Springer.

Lou, Y. \& LiU, D. (2010). Nano phosphide in weathering steels containing Ti by CSP processing. J Univ Sci Tech Beijing 32(9), $1163-1168$.

Vodarek, V., Cihal, V., Hubackova, J. \& Mazanec, K. (1988). Changes in precipitation and corrosion characteristics of 18/ $10 \mathrm{Ti}$ austenitic stainless steel exposed to prolonged annealing at $750-850^{\circ} \mathrm{C}$. Steel Res 59(11), 503-508. 